\title{
The crucial role of recovery capital in individuals with a gambling disorder
}

\author{
BELLE GAVRIEL-FRIED*
}

The Bob Shapell School of Social Work, Tel Aviv University, Ramat Aviv, Israel

(Received: September 11, 2017; revised manuscript received: February 22, 2018; second revised manuscript received: July 30, 2018; accepted: August 5, 2018)

\begin{abstract}
Background and aims: The concept of recovery capital $(\mathrm{RC})$ describes the internal and external resources that individuals draw upon to initiate and sustain the processes of addiction recovery. This concept has been primarily applied to individuals recovering from substance addictions. In this study, the RC concept was applied to individuals with a gambling disorder (GD) to test its associations with the diagnosis and severity of GD and with levels of psychopathology as manifested in depression and anxiety. Methods: A sample of 140 individuals who recovered or did not recover from a GD was drawn from lists of former and currently treated individuals in five gambling treatment centers in Israel. The DSM-5 diagnostic criteria for GD, Assessment of Recovery Capital and Brief Assessment of Recovery Capital Scales adapted to Gambling, Generalized Anxiety Disorder Scale, and the Patient Health Questionnaire 9 which measures depression were used. Results: RC clearly distinguishes between individuals who have recovered from GD and those who have not. A structural equation model showed significant negative associations between RC and GD severity, depression, and generalized anxiety. The associations between GD severity and depression and anxiety were not significant. However, when omitting the path between RC and depression/anxiety, the associations between GD and depression/anxiety became significant. Conclusions: RC plays an important role in GD severity and diagnosis, as well as in psychopathology. This study extends the concept of RC to the area of gambling and contributes to the growing body of studies that have found parallels and common denominators between substance addiction and behavioral addictions.
\end{abstract}

Keywords: gambling disorder, recovery, recovery capital

\section{INTRODUCTION}

A gambling disorder (GD) is defined as "persistent and recurrent problematic gambling behavior leading to clinically significant impairment or distress as indicated by the individual exhibiting four (or more) of (the diagnostic criteria) in a 12-month period" [fifth edition of the Diagnostic and Statistical Manual of Mental Disorders (DSM-5; American Psychiatric Association, 2013, p. 585)]. Nationally representative samples worldwide show that GD rates range from $0.1 \%$ to $2 \%$ (Petry, 2016).

There are known associations between symptoms of depression and anxiety disorders and GD (Lorains, Cowlishaw, \& Thomas, 2011; Petry, 2016). This has been found in national epidemiological studies (Kessler et al., 2008; Petry, Stinson, \& Grant, 2005) and in clinical samples of problem and pathological gamblers (Barrault, Bonnaire, \& Herrmann, 2017; Jauregui, Onaindia, \& Estévez, 2017). It has been suggested that these associations may be inherent to the notion that gambling is a maladaptive way of relieving depression and anxiety (Blaszczynski \& Nower, 2002; Jauregui et al., 2017). It is sometimes assumed that anxiety and depressive symptoms can increase in individuals with a GD as a result of the negative consequences of gambling behavior (Jauregui et al., 2017).
Recovery is a generic term indicative of adaptive changes in psychosocial functioning and/or a reduction in symptomatology and is one of the pillars of gambling treatment interventions (Nower \& Blaszczynski, 2008). Recovery has primarily been measured in terms of absence or decreases in gambling behaviors and GD symptoms (Slutske, Blaszczynski, \& Martin, 2009; Slutske, Piasecki, Blaszczynski, \& Martin, 2010; Toneatto et al., 2008) and other psychiatric manifestations. This is in line with studies that have explored the impact of psychological treatment of pathological gamblers (for a review, see Pallesen, Mitsem, Kvale, Johnsen, \& Molde, 2005). These studies define the outcome measures as decreases in key facets of gambling behaviors, such as the frequency of gambling, gambling urges, and $D S M-5$ disordered gambling symptoms, as well as associated psychiatric symptoms, such as depression and anxiety (Pickering, Keen, Enstwistle, \& Blaszczynski, 2017).

Several studies have examined pathways to recovery in individuals who have been both treated and untreated for gambling problems (Hodgins \& el-Guebaly, 2000;

* Corresponding address: Belle Gavriel-Fried, PhD; The Bob Shapell School of Social Work, Tel Aviv University, Ramat Aviv, Tel Aviv 69978, Israel; Phone: +972 3 6409131; Fax: +972 3 6409563, E-mail: bellegav@tauex.tau.ac.il

This is an open-access article distributed under the terms of the Creative Commons Attribution-NonCommercial 4.0 International License, which permits unrestricted use, distribution, and reproduction in any medium for non-commercial purposes, provided the original author and source are credited, a link to the CC License is provided, and changes - if any - are indicated. 
Hodgins, Wynne, \& Makarchuk, 1999; Slutske, 2006; Slutske et al., 2010; Toneatto et al., 2008). The findings show that most individuals diagnosed as disordered gamblers recover naturally without formal treatment (Hodgins et al., 1999; Slutske, 2006). Although recovered gamblers who are treated generally tend to have more severe gambling problems than those who recover naturally, similar recovery techniques (such as cognitive and behavioral strategies) are used by the two groups (Toneatto et al., 2008). Nevertheless, no study has explored the multiple human and social resources implemented by individuals who recover from GD to maintain their recovery.

One of the keys to understanding how individuals maintain momentum throughout the process of addiction recovery lies in the concept of recovery capital (RC; Cloud \& Granfield, 2001, 2008). RC emerged from observations of individuals who recovered from substance addictions and reflected a paradigm shift from an illness/crisis model to a model of recovery management. $\mathrm{RC}$ is conceptually linked to natural recovery, recovery management, resilience and protective factors, and the ideas of hardiness, wellness, and global health (Tew, 2013). According to Cloud and Granfield (2008), RC refers to the internal and external resources that individuals can draw upon to initiate and sustain processes of addiction recovery, which can be accumulated or exhausted over time, and is relevant to all stages of recovery. In their conceptual model, Cloud and Granfield (2008) suggested that RC has four main components: physical capital (e.g., income, savings, investments, and property), cultural capital (e.g., values, beliefs, and attitudes that promote social norms), human capital (e.g., education, knowledge, skills, hope, health, and heredity), and social capital (e.g., relationships, including family, friends, and broader social networks). More recently, a systematic literature review identified eight domains of $\mathrm{RC}$ relating to areas of physical, human, personal recovery, growth, health, social/ family, cultural, and community recovery (Hennessy, 2017).

RC has been examined extensively within the field of substance addiction, both in qualitative and quantitative studies. Qualitative studies have supported the claim that $\mathrm{RC}$ resources are crucial to the recovery process (Gueta \& Addad, 2015; Neale, Nettleton, \& Pickering, 2014). The results from quantitative studies suggest that individuals in recovery have the higher levels of RC than non-recoverers (Best, McKitterick, Beswick, \& Savic, 2015; Groshkova, Best, \& White, 2013; Laudet \& White, 2008).

GD has been included under the section on substancerelated and addictive disorders in the DSM-5 since 2013 (Straussner, 2013), based on evidence showing similarities between the two disorders in terms of biological dysfunctions, symptom presentations, and treatment approaches (Hasin et al., 2013; Petry, 2016). This points to the importance of determining whether the $\mathrm{RC}$, which is an emerging construct in the field of addiction (Keane, McAleenan, \& Barry, 2014) and is manifested in individuals who have recovered from substance disorders, is applicable to individuals who have recovered from GD as well.

The aim of this study was to apply the concept of RC to GD by (a) applying the Assessment of Recovery Capital (ARC) scale to GD and (b) to differentiate between individuals who have recovered from GD from those who have not recovered in levels of RC. It also delved deeper into the concept of RC to examine whether it is associated with GD severity and levels of psychopathology, as manifested in depression and anxiety. Two hypotheses were tested to assess whether the concept of $\mathrm{RC}$ could be associated with the diagnosis and severity of GD and the severity of psychopathological symptoms: (a) lower levels of RC would be found in individuals with active GD than in recovered individuals and (b) lower levels of RC would be associated with higher levels of GD severity and more elevated levels of depression and anxiety.

\section{METHODS}

\section{Procedures}

This study is part of a wider research project assessing $\mathrm{RC}$ in individuals with a GD. Individuals over the age of 18 years who reported lifetime DSM-5 GD without comorbid addiction to substances in the previous year (according to the DSM-5 criteria for drugs and alcohol) were considered eligible. The sample consisted of individuals who had either recovered or not recovered from a GD drawn from five outpatient gambling treatment centers in five large cities in Israel. The potential recovery period was limited to 1-5 years, a length of time that is considered medium-term recovery (Groshkova et al., 2013). The first appeal to individuals who were treated in these centers from 2011 to 2016 (both those who terminated or dropped out) and individuals in treatment during the study period was made by the administrative staff of the treatment centers. The names of those who had consented were given to the research team. A pilot test was conducted on five individuals who had recovered and five who had not recovered, which led to several changes in the questionnaire. The data were collected on tablets using Qualtrics software that allowed the principal investigator to monitor the data collection and make sure that the sample would consist of both recovered and non-recovered gamblers. There were no missing values in the data. The participants were compensated for their time in the form of a 100 Israeli Shekel (about 25.00 USD) shopping voucher. The data were collected between March and June 2017.

\section{Measures}

RC was measured by two scales. The data were collected using a modified version of the Assessment of Recovery Capital (Groshkova et al., 2013), which is most frequently used in the substance addiction field (Hennessy, 2017). It consists of a 50-item unidimensional scale measuring $\mathrm{RC}$ at different stages of recovery. The scale is conceptually divided into 10 domains, each of which is comprised of five items measuring recovery experience, meaningful activity, coping and life functioning, social support, global physical health, global psychological health, risk taking, housing and safety, meaningful activities, citizenship and community involvement, and substance use and sobriety. The ARC was developed for individuals who have recovered from substance disorders and for the purposes of this study was modified for GD such that (a) items related to substance use were reworded to refer to gambling behavior. For example, the item "I have more important things in my 
life than using substances" was changed to "I have more important things in my life than engaging in gambling." Two other modifications were made following the pilot test: (b) changing responses from binary to a 5-point Likert scale ranging from 1 (do not agree at all) to 5 (strongly agree) allowing for a neutral response and (c) asking participants to relate to the previous year rather than the day they filled in the questionnaire, to reflect the central premise that RC accumulates or exhausts over time (Skogens \& von Greiff, 2014). Higher ARC scores indicate higher RC. The Cronbach's $\alpha$ on the modified 50-item ARC was 95 .

The Brief Assessment of Recovery Capital (BARC-10). BARC-10 is an abbreviated version of the ARC scale containing 10 items measuring RC (Vilsaint et al., 2017). Each item represents one of the 10 conceptual domains from the original ARC measure (Groshkova et al., 2013). The BARC-10 (which was published during the data collection of this study) contains the ARC modifications described above. A higher $B A R C-10$ score indicates higher levels of $\mathrm{RC}$. The original reliability as reported by the authors was $\alpha=.90$, and in this study $\alpha=.75$.

DSM-5 diagnostic criteria for GD. This scale contains nine items assessing GD in the past 12 months. Individuals answered all items on a binary scale. Individuals with a score below 4 are considered non-disordered gamblers. For the purposes of this study, two scores were calculated: a binary diagnostic score measured as above or below the threshold of four DSM-5, reflecting correspondence to GD criteria (recovered vs. non-recovered), and a continuous score representing the severity of GD. The reliability of this measure was $\alpha=.91$.

Generalized Anxiety Disorder Scale (GAD-7). This sevenitem anxiety scale evaluates the presence and severity of GAD (Spitzer, Kroenke, Williams, \& Löwe, 2006). The respondents relate to the previous 2 weeks. Responses on each item range from 0 (not at all) to 3 (nearly every day), and the scores are summed. Higher GAD-7 scores indicate higher levels of anxiety. The reliability reported by the original authors was $\alpha=.92$. The Cronbach's $\alpha$ in this was .91 .

The Patient Health Questionnaire-9 (PHQ-9). This wellvalidated scale was developed by Kroenke and Spitzer (2002). Its nine items assess the severity of depression, based on DSM-IV criteria for depressive episodes. The respondents relate to the previous 2 weeks. Responses for each item range from 0 (not at all) to 3 (nearly every day). The total score ranges from 0 to 27 . Higher PHQ-9 scores indicate higher depression. The original reliabilities ranged from .89 to .84 . The Cronbach's $\alpha$ in this was .84 .

In addition, sociodemographic characteristics including gender, age, level of education, and marital status (married vs. unmarried) were collected. When necessary, the questionnaires were translated from English to Hebrew using the forward and backward translation procedures (Brisling, 1986).

\section{Participants}

The sample consisted of 140 individuals $(39 ; 27.9 \%$ women) aged 23-77 years $(M=49.15, S D=13.93)$, of whom 71 $(51.1 \%)$ were married. The mean number of years of education was $12.27(S D=1.93)$. Forty-nine individuals were classified as disordered gamblers (non-recovered) based on the DSM-5 GD criterion in the past year, and 91 individuals who were identified as recovered fell below the DSM-5 threshold.

\section{Statistical analyses}

Descriptive statistics are reported here in terms of the means and standard deviations. Hypotheses regarding the associations between the ARC score and the outcome measures (GD severity, depression, and anxiety) were tested with Pearson's correlation coefficients. In addition, an independent samples $t$-test was conducted to probe for differences between recovered and non-recovered gamblers (as measured by the DSM-5 criteria) in relation to the RC.

Using Mplus version 8 (Muthén \& Muthén, 1998-2012), confirmatory factor analysis (CFA) were performed on the ARC and BARC-10 scales. Then, a structural equation methodology was utilized to test for a comprehensive model with the associations of RC to both GD severity and psychopathology (depression and anxiety). Specifically, the RC score was simultaneously regressed on all of the outcome measures. Following the results accepted in the theoretically driven model, as well as the empirically found correlations, another alternative model was tested with the association between RC and GD severity omitted.

A sample size for structural equation model (SEM) analysis was calculated using the Preacher's method (Preacher \& Coffman, 2006) and was found suitable. Given its advantages, an item-parceling strategy was assigned randomly to create three indicators each for RC, DSM-5, and depression and anxiety (Coffman \& MacCallum, 2005; Landis, Beal, \& Tesluk, 2000; Thompson, 2005). Hence, groups of two items or more were aggregated through summing or averaging into subsets, which in turn were treated as indicators of the latent construct (Landis et al., 2000).

To estimate the CFA and SEM models' goodness-of-fit, three approximate fit indices were tested in addition to the $\chi^{2}$ statistic: the Tucker-Lewis index (TLI), the comparative fit index (CFI), and the root mean square error of approximation (RMSEA). A TLI and CFI close to 0.95 and RMSEA of 0.06 or lower reasonably indicate good fit $(\mathrm{Hu} \&$ Bentler, 1999). For the RMSEA index, values <.05 represent excellent model fit, 0.05-0.08 represent moderate fit, and 0.080.10 represent acceptable fit (Browne \& Cudeck, 1993).

\section{Ethics}

All procedures were reviewed and approved by the institutional review board of Tel Aviv University and the Ministry of Welfare Review Board. The study was conducted in accordance with the ethical standards of the American Psychological Association. All participants were informed of the aims of the study and they all provided informed written consent.

\section{RESULTS}

\section{Confirmatory factor analyses (CFAs)}

A CFA was conducted on the 50-item ARC based upon the original conceptual domains specified by 
Groshkova et al. (2013) but did not show an acceptable fit, $\chi^{2}(1,130, N=140)=2,422.59, p<.001$, TLI $=0.64$, $\mathrm{CFI}=0.67$, and RMSEA $=0.09[90 \%$ confidence interval $(\mathrm{CI})=0.09,0.10]$. Hence, for the purposes of this study, BARC-10, which is a brief version of the ARC, was used.

A CFA was performed on the BARC-10 for GD. Two items with low loadings were omitted ("I am proud of the community I live in and feel a part of it" and "I regard my life as challenging and fulfilling without the need to gamble"). Following the omission, the scale indicated good fit indices, $\chi^{2}(16, N=140)=30.45, p<.02$, TLI $=0.96$, $\mathrm{CFI}=0.98$, and RMSEA $=0.08[90 \% \mathrm{CI}=0.03,0.12]$. All the loading scores of the eight items ranged between 0.51 and 0.82 .

\section{Descriptive statistics and intercorrelations}

The means and standard deviations for the variables as well as their intercorrelations are presented in Table 1. This table shows that RC was negatively and significantly linked to the GD severity, depression, and anxiety. In addition, positive significant associations were found between GD severity and depression and anxiety, and a positive significant association was found between anxiety and depression. The sociodemographic variables (gender, age, education, and marital status) were not significantly associated with RC.

\section{Differences between recovered and non-recovered gamblers}

The differences between recovered and non-recovered gamblers (as measured by the DSM-5 criteria) in relation to the RC were tested. An independent samples $t$-test indicated that the level of RC among the recovered gamblers $(M=35.25, S D=3.95)$ was significantly higher than in the non-recovered group $(M=29.45, S D=6.36),[t(138)=$ $6.65, p<.001]$.

\section{Associations between $R C$ and $G D$ severity and depression and anxiety}

A SEM model of RC predicting the three dependent variables of GD severity, depression, and anxiety was tested and showed moderate fit indices, $\chi^{2}(48, N=140)=77.99$, $p=.004, \quad \mathrm{TLI}=0.97, \quad \mathrm{CFI}=0.97$, and $\mathrm{RMSEA}=0.07$ $[90 \% \mathrm{CI}=0.04,0.09]$. The four sociodemographic variables (age, gender, education, and marital status) were added to the initial model as covariates of RC. Since none of these covariates were significantly related to any of the dependent variables, they were omitted from the analysis. Because the resulting model was equivalent to the original model, its fit indices were the same as reported above. In this model, the paths from $\mathrm{RC}$ to the three dependent variables of GD severity, depression, and anxiety were significant (all $p$ 's $<$ $.001)$. Negative associations were found between RC and GD severity, between RC and depression, and between RC and anxiety (Figure 1).

However, the model yielded no significant associations between GD severity and depression and anxiety. This finding was surprising given the widely documented theoretical and empirical evidence. Specifically, the correlations reported in this study showed significant positive correlations between these factors and that these associations were theoretically driven. Hence, it was decided to test for a model in which the association between RC and depression/ anxiety were omitted. The rationale was to test the variance explained in depression/anxiety by GD severity without the effect of RC on depression/anxiety. The model showed acceptable fit indices, $\chi^{2}(48, N=140)=124.92, \quad p=$ $0.000, \mathrm{TLI}=0.92, \mathrm{CFI}=0.94$, and $\mathrm{RMSEA}=0.09[90 \%$ $\mathrm{CI}=0.08,0.11]$. In the model (shown in Figure 2), it was evident that RC was negatively associated with GD severity. GD severity was positively associated with depression and anxiety. In both models, a significant correlation was found between depression and anxiety.

\section{DISCUSSION AND CONCLUSIONS}

This is the first study to apply the concept of RC to individuals with a GD. The main findings highlight the pivotal role of $\mathrm{RC}$ in relation to both a rigorous diagnosis of GD and more subtle measure of gambling severity. The analyses revealed high correlations between RC and GD severity and levels of depression and anxiety. Furthermore, $\mathrm{RC}$ was shown to successfully distinguish between individuals with an active GD and recovered gamblers. These findings are consistent with a study conducted by Best et al. (2015), who found that individuals who describe themselves as completely abstinent from substances had higher levels of $\mathrm{RC}$ and fewer symptoms of depression and generalized anxiety than those who had not recovered. The strength of this study thus lies in basing the GD diagnosis on solid DSM-5 criteria and in the usage of a continuous measure of GD severity. This should encourage clinicians to identify patients who do not directly correspond to all the criteria of GD but still display gambling problems reflected in the subclinical population.

The study aimed to suggest a theoretical comprehensive model that includes the associations between RC and GD severity on the one hand and between RC and depression and anxiety on the other hand. The central theoretical model

Table 1. Means, standard deviations $(S D \mathrm{~s})$, and intercorrelations $(N=140)$

\begin{tabular}{lrcccc}
\hline Variable & Mean & $S D$ & 1 & 2 & 3 \\
\hline 1. Recovery capital & 33.22 & 5.64 & - & & \\
2. Gambling disorder severity & 2.56 & 3.10 & $-.47^{* * *}$ & - & \\
3. Depression & 5.99 & 5.69 & $-.64^{* * *}$ & $.49^{* * *}$ & - \\
4. Anxiety & 4.76 & 5.44 & $-.47^{* * *}$ & $.40^{* * *}$ & $.82^{* * *}$ \\
\hline Note. ${ }^{* * *} p<.001$. & & & & &
\end{tabular}




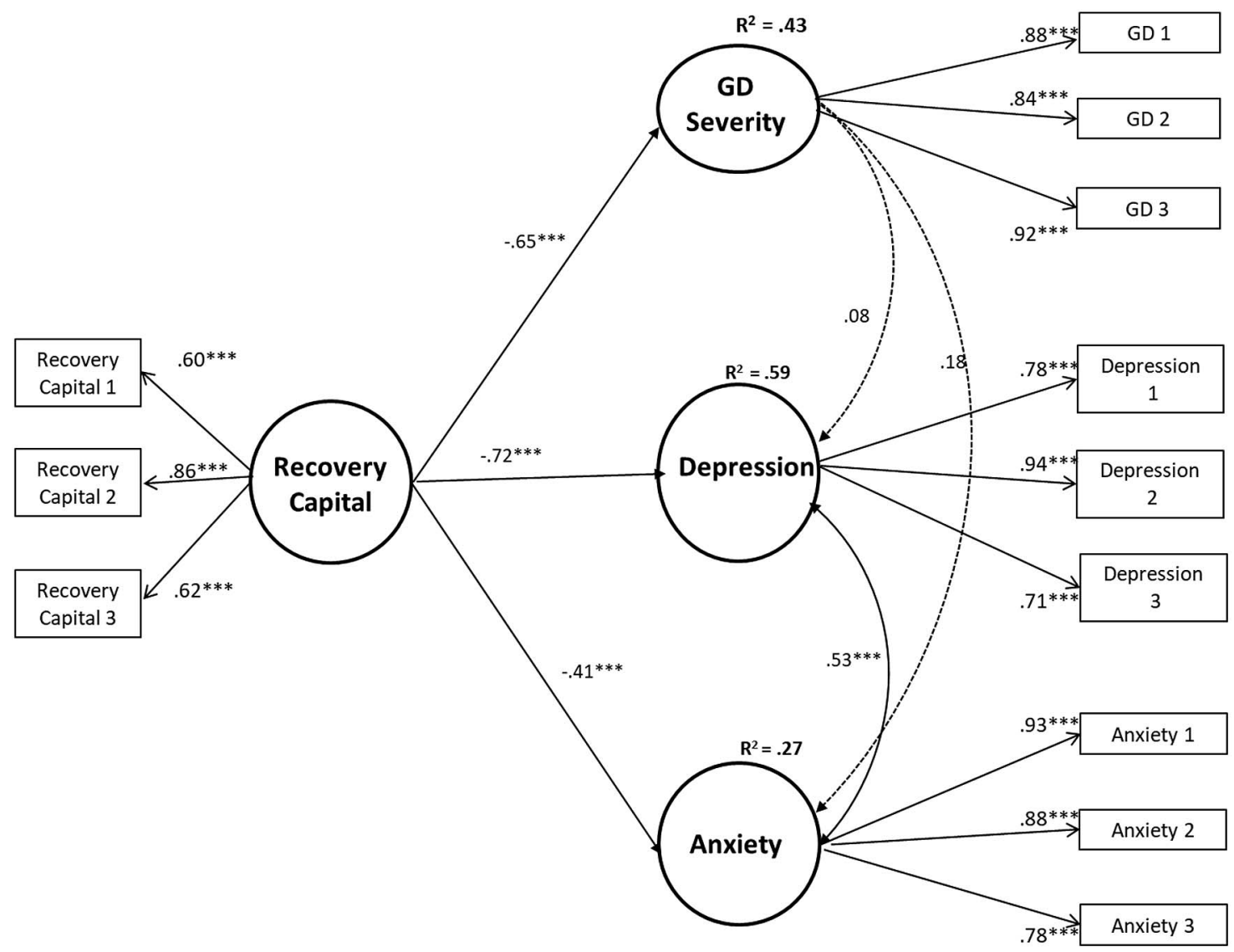

Figure 1. Structural equation model of the relationship between recovery capital and gambling disorder (GD) severity, depression and anxiety with standardized parameters. The solid lines indicate paths that were statistically significant at $p<.001$ and the dashed lines correspond to non-significant correlations. $* * * p<.001$

showed that RC is negatively associated with GD severity and with depression and anxiety - both of which are known to be common in individuals with an active GD (Nower \& Blaszczynski, 2008; Petry et al., 2005). However, the theoretically expected associations between GD severity and depression and anxiety were not significant. These results were surprising, given the significant correlations reported in this study and previous studies that have reported an association between problem or pathological gambling and depression and anxiety (Barrault et al., 2017; Jauregui et al., 2017; Kessler et al., 2008; Petry, 2016). Hence, the model needed to be tested thoroughly. An alternative model in which the paths between RC and depression and anxiety were omitted from the model indicated that GD severity was associated with higher levels of depression and anxiety.

Apparently, in the original model, GD severity did not have a significant link to anxiety/depression as a result of the $\mathrm{RC}$ factor, which accounted for the majority of explained variance in anxiety/depression. In other words, RC and gambling severity "competed" for the association with depression and anxiety, and thus making it impossible for both to be significant in one comprehensive model. This highlights the crucial role of RC in the model.

In this study, the conceptual construct of RC was applied to GD by administering the modified ARC scale, which explores 10 domains of RC. The CFA indicated poor model fit, which is congruent with previous use of the scale in individuals with substance disorders (Arndt, Sahker, \&
Hedden, 2017; Groshkova et al., 2013). For this reason, the shorter derivative version of this scale (BARC-10) developed by Vilsaint et al. (2017) that measures a single unified dimension of RC was applied here and was found to have an excellent fit. The measurement of $\mathrm{RC}$ on a single factor scale is consistent with the argument that the recovery process is a continuous one in which individuals possess and implement many domains of recovery resources that merge into one, thus justifying the claim that all these domains should be considered together (Hennessy, 2017).

However, despite the fact that this study used a unified construct of $\mathrm{RC}$, it is important to recall that the BARC scale was administered based on the validity and preliminary results of the ARC scale. This cannot negate the fact that other RC domains are relevant to GD even though they were not identified in this study by using quantitative methods. Future studies should work toward identifying these RC domains by applying qualitative research methods. In addition, a measure should be developed that can differentiate between different RC domains to help individuals and clinicians map the recovery resources in each individual, so that a suitable therapy program can be tailored to strengthen the domains pertinent to each. This type of tool could also help identify the RC domains characteristic of each type of addiction (behavioral addictions vs. substance addictions).

The findings have a practical application for therapy in which raising the levels of $\mathrm{RC}$ in individuals with a GD 


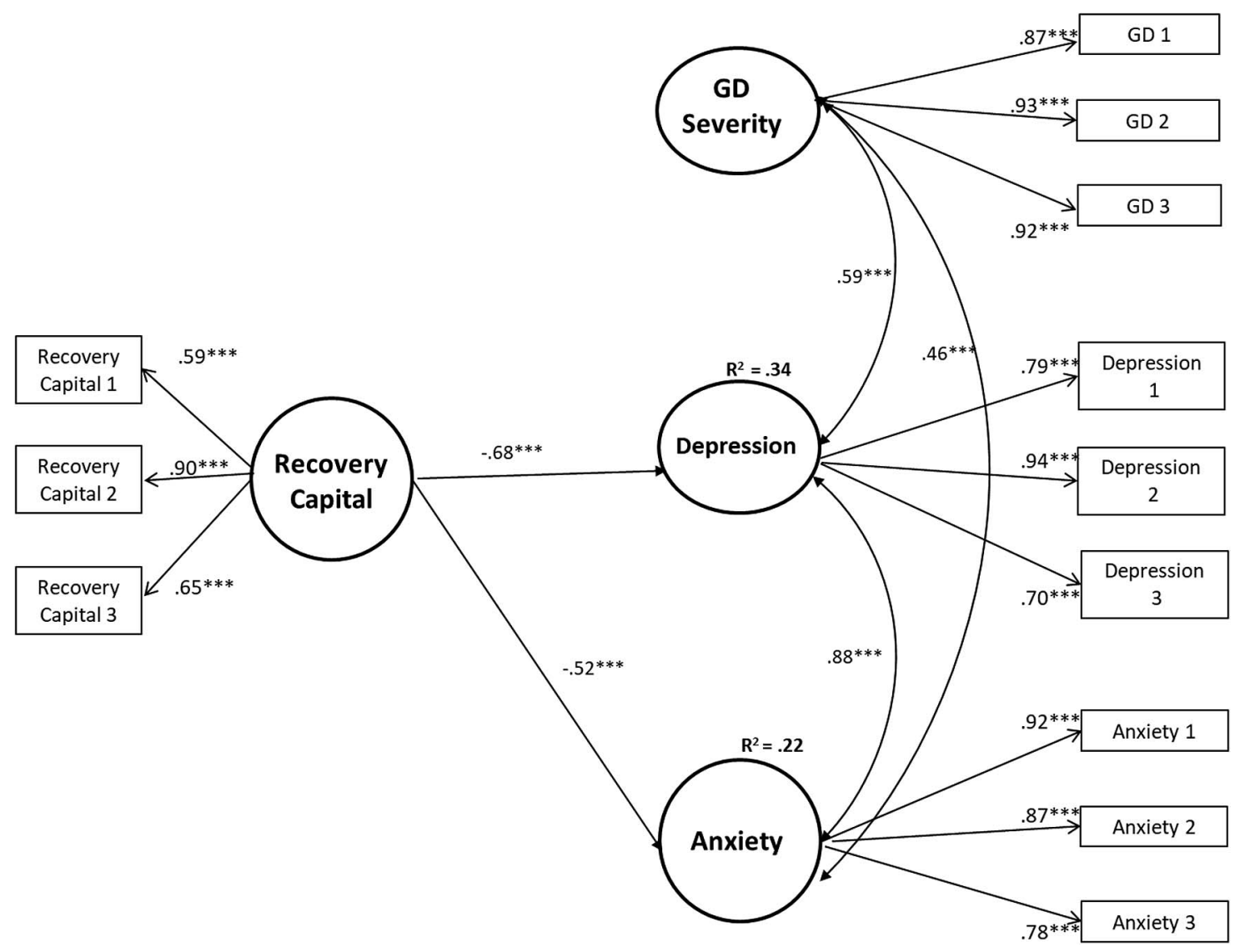

Figure 2. Alternative model of relationship between recovery capital and depression and anxiety, as well as gambling disorder (GD) severity and depression and anxiety with standardized parameters. The solid lines indicate paths that were statistically significant at $p<.001$ and the dashed lines correspond to non-significant correlations. ${ }^{* * *} p<.001$

might decrease the levels of gambling severity and levels of depression and anxiety. In a wider context, these findings stress the need for a paradigmatic shift away from the models of illness and pathology to a model of recovery management that enhances the RC among individuals with GD. Although for many years now the emphasis in therapy has been on restrictions and removal of risk factors from the lives of people with addiction problems, it is time to consider adding other resources to the mix (Tew, 2013) to enrich the lives of individuals with a GD on the personal, cultural, and social levels to favor the recovery process.

This study has several limitations. First, it was based on a convenience sample of gamblers who had sought treatment. Although such subjects typically have a more severe gambling problem than natural recoverers (Toneatto et al., 2008), it is reasonable to assume that they also have resources and strengths that enable them to ask for treatment. Further studies should be conducted on the RC of natural recoverers in relation to gambling. Second, the findings were based on self-reports with no cross-referencing with other sources. Finally, this was a cross-sectional study and by nature cannot provide indications on the causal links between the factors assessed.

Notwithstanding these limitations, this is the first study to apply the concept of RC, which has primarily been used to individuals with substance disorders, to individuals with a GD as defined by DSM- 5 criteria. It thus contributes to the growing body of studies that have found parallels and common denominators between substance addiction and behavioral addictions (Hasin et al., 2013; Petry, 2016). Thus, overall, this study helps to fill a gap in the theoretical and practical literature and enhances the generalizability of this important concept to individuals recovering from GD.

Funding sources: The study was funded by seed grant awarded to the author by the National Center for Responsible Gaming in 2017.

Author's contribution: BG-F is the sole author of this article. She had access to all the data from the study, both what is reported and what is unreported, and also that she had complete freedom to direct its analysis and its reporting, without influence from the sponsors. She declares that there was no editorial direction or censorship from the sponsors.

Conflict of interest: The author declares no conflict of interest from any kind.

Acknowledgements: The author gratefully acknowledges Yasmin Alkaly and Yafit Levin for their useful statistical advice. 


\section{REFERENCES}

American Psychiatric Association. (2013). Diagnostic and statistical manual of mental disorders, (5th ed.). Washington, DC: American Psychiatric Association.

Arndt, S., Sahker, E., \& Hedden, S. (2017). Does the Assessment of Recovery Capital scale reflect a single or multiple domains? Substance Abuse and Rehabilitation, 8, 39-43. doi:10.2147/ SAR.S138148

Barrault, S., Bonnaire, C., \& Herrmann, F. (2017). Anxiety, depression and emotion regulation among regular online poker players. Journal of Gambling Studies, 33(4), 1039-1050. doi:10.1007/s10899-017-9669-3

Best, D., McKitterick, T., Beswick, T., \& Savic, M. (2015). Recovery capital and social networks among people in treatment and among those in recovery in York, England. Alcoholism Treatment Quarterly, 33(3), 270-282. doi:10. 1080/07347324.2015.1050931

Blaszczynski, A., \& Nower, L. (2002). A pathways model of problem and pathological gambling. Addiction, 97(5), 487-499. doi:10.1046/j.1360-0443.2002.00015.x

Brisling, R. W. (1986). The wording and translation of research instruments. In W. J. Lonner \& J. W. Berry (Eds.), Field methods in cross-cultural research (pp. 137-164). Newbury Park, CA: Sage.

Browne, M. W. \& R. Cudeck (1993). Alternative ways of assessing model fit. In K. A. Bollen \& J. S. Long (Eds.), Testing structural equation models (pp. 136-162). Newbury Park, CA: Sage.

Cloud, W., \& Granfield, R. (2001). Natural recovery from substance dependency: Lessons for treatment providers. Journal of Social Work Practice in the Addictions, 1(1), 83-104. doi:10.1300/J160v01n01_07

Cloud, W., \& Granfield, R. (2008). Conceptualizing recovery capital: Expansion of a theoretical construct. Substance Use \& Misuse, 43(12-13), 1971-1986. doi:10.1080/10826080 802289762

Coffman, D. L., \& MacCallum, R. C. (2005). Using parcels to convert path analysis models into latent variable models. Multivariate Behavioral Research, 40(2), 235-259. doi:10.1207/s15327906mbr4002_4

Groshkova, T., Best, D., \& White, W. (2013). The Assessment of Recovery Capital: Properties and psychometrics of a measure of addiction recovery strengths. Drug and Alcohol Review, 32(2), 187-194. doi:10.1111/j.1465-3362.2012.00489.x

Gueta, K., \& Addad, M. (2015). A house of cards: The long-term recovery experience of former drug-dependent Israeli women. Women's Studies International Forum, 48, 18-28. doi:10.1016/j.wsif.2014.10.003

Hasin, D. S., O'Brien, C. P., Auriacombe, M., Borges, G., Bucholz, K., Budney, A., Compton, W. M., Crowley, T., Ling, W., Petry, N. M., Schuckit, M., \& Grant, B. F. (2013). DSM-5 criteria for substance use disorders: Recommendations and rationale. American Journal of Psychiatry, 170(8), 834-851. doi:10.1176/appi.ajp.2013.12060782

Hennessy, E. A. (2017). Recovery capital: A systematic review of the literature. Addiction Research \& Theory, 25(5), 349-360. doi:10.1080/16066359.2017.1297990

Hodgins, D. C., \& el-Guebaly, N. (2000). Natural and treatmentassisted recovery from gambling problems: A comparison of resolved and active gamblers. Addiction, 95(5), 777-789. doi:10.1046/j.1360-0443.2000.95577713.x
Hodgins, D. C., Wynne, H., \& Makarchuk, K. (1999). Pathways to recovery from gambling problems: Follow-up from a general population survey. Journal of Gambling Studies, 15(2), 93-104. doi:10.1023/A:1022237807310

Hu, L., \& Bentler, P. M. (1999). Cutoff criteria for fit indexes in covariance structure analysis: Conventional criteria versus new alternatives. Structural Equation Modeling, 6(1), 1-55. doi:10.1080/10705519909540118

Jauregui, P., Onaindia, J., \& Estévez, A. (2017). Adaptive and maladaptive coping strategies in adult pathological gamblers and their mediating role with anxious-depressive symptomatology. Journal of Gambling Studies, 33(4), 1081-1097. doi:10.1007/s10899-017-9675-5

Keane, M., McAleenan, G., \& Barry, J. (2014). Addiction recovery: A contagious paradigm! A case for the re-orientation of drug treatment services and rehabilitation services in Ireland. Dublin, Ireland: Solise. Retrieved from http://www. drugs.ie/resourcesfiles/ResearchDocs/Ireland/2014/Addiction RecoveryAContagiousParadigm.pdf

Kessler, R. C., Hwang, I., LaBrie, R., Petukhova, M., Sampson, N. A., Winters, K. C., \& Shaffer, H. J. (2008). DSM-IV pathological gambling in the National Comorbidity Survey Replication. Psychological Medicine, 38(9), 1351-1360. doi:10.1017/S0033291708002900

Kroenke, K., \& Spitzer, R. L. (2002). The PHQ-9: A new depression diagnostic and severity measure. Psychiatric Annals, 32(9), 509-515. doi:10.3928/0048-5713-20020901-06

Landis, R. S., Beal, D. J., \& Tesluk, P. E. (2000). A comparison of approaches to forming composite measures in structural equation models. Organizational Research Methods, 3(2), 186-207. doi:10.1177/109442810032003

Laudet, A. B., \& White, W. L. (2008). Recovery capital as prospective predictor of sustained recovery, life satisfaction, and stress among former poly-substance users. Substance Use \& Misuse, 43(1), 27-54. doi:10.1080/10826080701681473

Lorains, F. K., Cowlishaw, S., \& Thomas, S. A. (2011). Prevalence of comorbid disorders in problem and pathological gambling: Systematic review and meta-analysis of population surveys. Addiction, 106(3), 490-498. doi:10.1111/j.1360-0443.2010. 03300.x

Muthén, L. K., \& Muthén, B. O. (1998-2012). Mplus user's guide (7th ed.). Los Angeles, CA: Muthén \& Muthén.

Neale, J., Nettleton, S., \& Pickering, L. (2014). Gender sameness and difference in recovery from heroin dependence: A qualitative exploration. International Journal of Drug Policy, 25(1), 3-12. doi:10.1016/j.drugpo.2013.08.002

Nower, L., \& Blaszczynski, A. (2008). Recovery in pathological gambling: An imprecise concept. Substance Use \& Misuse, 43(12-13), 1844-1864. doi:10.1080/10826080802285810

Pallesen, S., Mitsem, M., Kvale, G., Johnsen, B. H., \& Molde, H. (2005). Outcome of psychological treatments of pathological gambling: A review and meta-analysis. Addiction, 100(10), 1412-1422. doi:10.1111/j.1360-0443.2005.01204.x

Petry, N. (2016). Behavioral addictions: DSM-5 and beyond. London, UK: Oxford University Press.

Petry, N. M., Stinson, F. S., \& Grant, B. F. (2005). Comorbidity of DSM-IV pathological gambling and other psychiatric disorders: Results from the National Epidemiologic Survey on Alcohol and Related Conditions. The Journal of Clinical Psychiatry, 66(5), 564-574. doi:10.4088/JCP. v66n0504 
Pickering, D., Keen, B., Enstwistle, G., \& Blaszczynski, A. (2017). Measuring treatment outcomes in gambling disorders: A systematic review. Addiction, 113(3), 411-426. doi:10.1111/ add. 13968

Preacher, K. J., \& Coffman, D. L. (2006). Computing power and minimum sample size for RMSEA [Computer software]. Retrieved from http://quantpsy.org/

Skogens, L., \& von Greiff, N. (2014). Recovery capital in the process of change - Differences and similarities between groups of clients treated for alcohol or drug problems. European Journal of Social Work, 17(1), 58-73. doi:10.1080/ 13691457.2012 .739559

Slutske, W. S. (2006). Natural recovery and treatment-seeking in pathological gambling: Results of two US national surveys. American Journal of Psychiatry, 163(2), 297-302. doi:10.1176/appi.ajp.163.2.297

Slutske, W. S., Blaszczynski, A., \& Martin, N. G. (2009). Sex differences in the rates of recovery, treatment-seeking, and natural recovery in pathological gambling: Results from an Australian community-based twin survey. Twin Research and Human Genetics, 12(5), 425-432. doi:10.1375/twin.12.5.425

Slutske, W. S., Piasecki, T. M., Blaszczynski, A., \& Martin, N. G. (2010). Pathological gambling recovery in the absence of abstinence. Addiction, 105(12), 2169-2175. doi:10.1111/ j.1360-0443.2010.03080.x
Spitzer, R. L., Kroenke, K., Williams, J. B., \& Löwe, B. (2006). A brief measure for assessing generalized anxiety disorder: The GAD-7. Archives of Internal Medicine, 166(10), 1092-1097. doi:10.1001/archinte.166.10.1092

Straussner, S. L. A. (2013). The DSM-5 diagnostic criteria: What's new? Journal of Social Work Practice in the Addictions, 13(4), 448-453. doi:10.1080/1533256X.2013.840199

Tew, J. (2013). Recovery capital: What enables a sustainable recovery from mental health difficulties? European Journal of Social Work, 16(3), 360-374. doi:10.1080/13691457. 2012.687713

Thompson, J. A. (2005). Proactive personality and job performance: A social capital perspective. Journal of Applied Psychology, 90(5), 1011-1017. doi:10.1037/0021-9010.90. 5.1011

Toneatto, T., Cunningham, J., Hodgins, D., Adams, M., Turner, N., \& Koski-Jannes, A. (2008). Recovery from problem gambling without formal treatment. Addiction Research \& Theory, 16(2), 111-120. doi:10.1080/16066350801923638

Vilsaint, C. L., Kelly, J. F., Bergman, B. G., Groshkova, T., Best, D., \& White, W. (2017). Development and validation of a Brief Assessment of Recovery Capital (BARC-10) for alcohol and drug use disorder. Drug and Alcohol Dependence, 177, 71-76. doi:10.1016/j.drugalcdep.2017.03.022 